\title{
Boolean algebras in which every chain and antichain is countable
}

\section{by}

J. E. Baumgartner* (Hanover, N. H.) and P. Komjáth (Budapest)

Abstract. Using the combinatorial axion $\diamond$, we deduce the existence of uncountable Boolean algebras in which every chain and antichain is countable. We find examples of such algebras for which the automorphism group has either 1 or $2^{\aleph_{0}}$ elements, and we apply a result of Kunen and Tall to conclude that the existence of such algebras is relatively consistent with the negation of the continuum hypothesis.

We also prove that if $B$ is a Boolean algebra in which every antichain is countable then $B$ has a countable dense subalgebra, and if $B$ is atomless then the automorphism group of $B$ has either 1 or $2^{\mathrm{N}_{0}}$ elements.

0. Introduction. A chain in a Boolean algebra is a set which is linearly ordered by the canonical partial ordering associated with the algebra; an antichain is a set of pairwise incomparable elements of the algebra. Some authors use the word "antichain" to denote a set of pairwise disjoint elements; note that this is not the case here.

Using the combinatorial axiom $\gamma$, we deduce the existence of uncountable Boolean algebras in which every chain and antichain is countable. We find examples of such algebras for which the automorphism group has either 1 or $2^{\mathrm{No}}$ elements, and we apply a result of Kunen and Tall to conclude that the existence of such algebras is relatively consistent with the negation of the continuum hypothesis.

We also prove that if $B$ is a Boolean algebra in which every antichain is countable then $B$ has a countable dense subalgebra, and if $B$ is atomless then the automorphism group of $B$ has either 1 or $2^{\mathrm{No}}$ elements.

Our set-theoretic terminology is standard. If $C \subseteq \omega_{1}$, then $C$ is closed unbounded iff $\forall \alpha<\omega_{1} \exists \beta \in C \alpha \leqslant \beta$ and $\forall \alpha<\omega_{1} \sup (C \cap \alpha) \in C$. If $S \subseteq \omega_{1}, S$ is stationary iff $S \cap C \neq 0$ for every closed unbounded set $C$. Fodor's Theorem [4] asserts that if $S$ is stationary, $f S \rightarrow \omega_{1}$, and $f(\alpha)<\alpha$ for all $\alpha \in S$, then there is stationary $S^{\prime} \subseteq S$ such that $f$ is constant on $S^{\prime}$.

A set mapping on a set $X$ is a function $f$ such that for all $x \in X, f(x) \subseteq X-\{x\}$. A set $Y \subseteq X$ is free (with respect to $f$ ) iff $\forall y, z \in Y z \notin f(y)$. Hajnal (see [5] or

* Research partially supported by National Science Foundation grant MCS 76-08231. 
$[3, \S 44])$ has proved that if $X$ has cardinality $x, f(x)$ has cardinality $<\lambda$ for all $x \in X$, and $\lambda<x$, then there is a free set of cardinality $x$. We will need this result in the next section for $x=\kappa_{2}$ and $\lambda=s_{1}$.

The proposition $\diamond$ asserts that there is a sequence $\left\langle S_{\alpha}: \alpha\left\langle\omega_{1}\right\rangle\right.$ such that $S_{\alpha} \subseteq \alpha$ and for any $S \subseteq \omega_{1},\left\{\alpha: S \cap \alpha=S_{\alpha}\right\}$ is stationary. We refer to $\left\langle S_{\alpha}: \alpha<\omega_{1}\right\rangle$ as a $\diamond$-sequence. Jensen has shown that $\diamond$ is true in $L$, the universe of constructible sets. See [2].

1. Statement of results. An element $b$ of a Boolean algebra $B$ will be called uncountable iff $\{a \in B: a \leqslant b\}$ is uncountable; otherwise $b$ is countable.

The main results of the paper are the following:

THEOREM 1. Assume $\diamond$. Then there is an atomless field of subsets of $\omega$ such that every nonzero element is uncountable and every chain and antichain is countable.

THEOREM 2. Assume $\diamond$. Then there is an uncountable atomless field of subsits of $\omega$ such that the countable elements form a maximal ideal and every antichain (and hence every chain) is countable.

Theorems 1 and 2 are proved in Sections 2 and 3, respectively.

The next theorem shows that it is no accident that fields of subsets of $\omega$ are involved in Theorems 1 and 2.

A subalgebra $D$ of a Boolean algebra $B$ is dense in $B$ iff for every nonzero $b \in B$ there is nonzero $d \in D$ such that $d \leqslant b$.

THEOREM 3. Let $B$ be a Boolean algebra in which every antichain is countable. Then $B$ has a countable dense subalgebra, and hence $B$ is representable as a field of subsets of $\omega$.

Pro of. Suppose not. Then it is easy to obtain a sequence $\left\langle b_{\alpha}: \alpha<\omega_{1}\right\rangle$ of nonzero elements of $B$ such that for each $\alpha$, if $B_{\alpha}$ is the subalgebra of $B$ generated by $\left\{b_{\beta}: \beta<\alpha\right\}$, then there is no nonzero $d \in B_{\alpha}$ such that $d \leqslant b_{\alpha}$. Let

$$
Z=\left\{\alpha<\omega_{1}: \exists b \in B_{\alpha} b \wedge b_{\alpha}, b_{\alpha}-b \neq 0\right\} .
$$

Case 1. $Z$ is stationary. Let $\left\langle c_{\alpha}: \alpha<\omega_{1}\right\rangle$ enumerate $\bigcup\left\{B_{\alpha}: \alpha<\omega_{1}\right\}$ and for each $\alpha \in Z$ let $f(\alpha)$ be the least ordinal $\beta$ such that $b_{\alpha} \wedge c_{\beta}, b_{\alpha}-c_{\beta} \neq 0$. Let $C=\left\{\alpha: B_{\alpha}=\left\{c_{\beta}: \beta<\alpha\right\}\right\}$. It is clear that $C$ is closed unbounded, so $C \cap Z$ is stationary. Moreover, $f(\alpha)<\alpha$ for every $\alpha \in C \cap Z$. By Fodor's Theorem there is stationary $S \subseteq C \cap Z$ on which $f$ is constant.

Let $c=c_{f(\alpha)}$, where $\alpha \in S$ is arbitrary. For each $\alpha \in S$ let $d_{\alpha}=\left(c \wedge b_{\alpha}\right) \vee\left(\bar{c} \wedge \bar{b}_{\alpha}\right)$ ( $\bar{c}$ denotes the complement of $c$ ). Then we claim $\left\{d_{\alpha}: \alpha \in S\right\}$ is an uncountable set of pairwise incomparable elements, contradiction. Suppose $\alpha, \beta \in S$ and $\alpha<\beta$. If $d_{\alpha} \leqslant d_{\beta}$, then $c \wedge b_{\alpha} \leqslant b_{\beta}$ and $c \wedge b_{\alpha} \in B_{\beta}$, contradicting the choice of $b_{\beta}$. If $d_{\beta} \leqslant d_{\alpha}$ then $\bar{c} \wedge \bar{b}_{\beta} \leqslant \bar{c} \wedge \bar{b}_{\alpha}$ so $\bar{c} \wedge b_{\alpha} \leqslant \bar{c} \wedge b_{\beta} \leqslant b_{\beta}$ and we reach a similar contradiction.

Case 2. $Z$ is nonstationary. Then $Y=\omega_{1}-Z$ is uncountable, and if $\alpha, \beta \in Y$ and $\alpha<\beta$ then either $b_{\alpha} \wedge b_{\beta}=0$ or $b_{\beta} \leqslant b_{\alpha}$.

Let $W=\left\{\alpha \in Y:\left\{\beta \in Y: b_{\beta} \leqslant b_{\alpha}\right\}\right.$ is uncountable $\}$. If $W$ is countable, then we construct $\left\langle b_{\alpha_{\xi}}: \xi<\omega_{1}\right\rangle$ inductively so that $\alpha_{\xi} \in Y-W$ and $b_{\alpha_{\xi}} \nless b_{\alpha_{\eta}}$ for all $\eta<\xi$.
But then $\left\{b_{\alpha \xi}: \xi<\omega_{1}\right\}$ is an uncountable pairwise disjoint set. Hence $W$ is uncountable.

If $\left\{b_{\alpha}: \alpha \in W\right\}$ is a chain then let $\left\langle c_{\alpha}: \alpha<\omega_{1}\right\rangle$ enumerate $\left\{b_{\alpha}: \alpha \in W\right\}$ in decreasing order (i.e. $\alpha<\beta \Rightarrow c_{\beta}<c_{\alpha}$ ). Then $\left\{c_{\alpha}-c_{\alpha+1}: \alpha<\omega_{1}\right\}$ is an uncountable pairwise disjoint set, contradiction. Hence $\left\{b_{\alpha}: \alpha \in W\right\}$ is not a chain.

It follows that there are $\alpha, \beta \in W$ with $b_{\alpha} \wedge b_{\beta}=0$. Now let

$$
f: \omega_{1} \rightarrow\left\{\gamma \in Y: b_{\gamma} \leqslant b_{\alpha}\right\}
$$

and $g: \omega_{3} \rightarrow\left\{\gamma \in Y: b_{\gamma} \leqslant b_{\beta}\right\}$ be one-to-one and such that if $\xi<\eta$ then $f(\xi), g(\xi)$ $<f(\eta), g(\eta)$. For each $\xi$ let $d_{\xi}=b_{f(\xi)} \vee\left(b_{\beta}-b_{g(\xi)}\right)$. Then if $\xi \neq \eta$ it is easy to check that $d_{\xi}$ and $d_{\eta}$ are incomparable. This contradiction completes the proof that $B$ has a countable dense subalgebra.

Since any countable Boolean algebra is representable as a field of subsets of $\omega$, the second assertion follows.

It should be remarked that this argument generalizes almost verbatim to larger cardinals.

It follows from Theorem 3 that every Boolean algebra with no uncountable antichains must have cardinality $\leqslant 2^{\mathrm{ko}}$. Must every uncountable such algebra have cardinality $\kappa_{1}$ ? Without using the continuum hypothesis we have been unable to settle this question, but we can say that the number of countable elements is not too large.

THEOREM 4. Suppose $B$ is a Boolean algebra in which every antichain has cardinality $\leqslant \kappa_{1}$. Then $B$ has at most $\kappa_{1}$ countable elements.

Proof. Suppose $X$ were a set of $s_{2}$ countable elements of $B$. For each $b \in X$, let $f(b)=\{c \in X: c<b\}$. By Hajnal's set-mapping theorem there is a free set $Y \subseteq X$ of cardinality $\aleph_{2}$. But the elements of $Y$ are clearly pairwise incomparable, contradiction.

A Boolean algebra is rigid if it has no automorphisms except the identity, THEOREM 5. Let $B$ be a Boolean algebra in which every antichain is countable.

(a) If every nonzero element of $B$ is uncountable, then $B$ is rigid.

(b) If $B$ is atomless and $B$ contains a nonzero countable element, then $B$ has exactly $2^{\text {so }}$ automorphisms.

Proof. (a) If $B$ had a nontrivial automorphism $f$, then there would be nonzero $b \in B$ such that $f(b) \wedge b=0$. But then $\{c \vee(f(b)-f(c)): c<b\}$ would be an uncountable set of pairwise incomparable elements, contradiction.

(b) Any countable atomless Boolean algebra has $2^{\text {so }}$ automorphisms so if $b \in B$ is nonzero and countable, there are at least $2^{\mathrm{N}_{0}}$ automorphisms of $B$ fixing $b$. On the other hand, by Theorem $3, B$ has a countable dense subalgebra $D$. Every automorphism of $B$ is completely determined by its values on $D$, and there are only $2^{\mathrm{k}_{0}}$ possible functions mapping $D$ into $B$.

COROLLARY 6. Assuming $\diamond$, there are uncountable Boolean algebras with no 3 - Fundamenta Mathematicae CXI. 2 - 
uncountable chains or antichains which are rigid, and there are such algebras with the: property that every uncountable atomless subalgebra has exactly $2^{\text {so }}$ automorphisms.

Finally, we observe that by a result of Kunen and Tall [7, Theorem 11], if $B$ is a Boolean algebra with no uncountable chain or antichain then $B$ remains such an: algebra in any extension of the universe via a property $(K)$ notion of forcing. Since the continuum hypothesis may be violated by a property $(K)$ notion of forcing (by adding many Cohen reals, for example), the conulusions of Theorems 1 and 2 and Corollary 6 are relatively consistent with the negation of the continuum hypothesis.

Several problems remain.

Problem 1. Can our uses of $\diamond$ be replaced by $\mathrm{CH}$ ? Using $\mathrm{CH}$, E. S. Berney [1] has constructed an uncountable Boolean algebra with no uncountable antichains, but his algebra has uncountable chains.

PRoBLEM 2. Is it consistent that every uncountable Boolean algebra has an uncountable antichain? It is conceivable that Martin's Axiom implies that there is no uncountable collection of subsets of $\omega$ in which all chains and antichains (with respect to inclusion) are countable. Kunen [6] (and the first author, indepen-. dently) used $\mathrm{CH}$ to construct a sequence $\left\langle A_{\alpha}: \alpha<\omega_{1}\right\rangle$ of subsets of $\omega$ such that if $\alpha<\beta$ then $A_{\beta}-A_{\alpha}$ is finite and $\left\{A_{\alpha}: \alpha<\omega_{1}\right\}$ has no uncountable chains or antichains, and Kunen proved that under Martin's Axiom no such sequence exists, but that. is the best result to date.

Problem 3. Is it provable in ZFC that every uncountable Boolean algebra. with no uncountable antichains has cardinality $\kappa_{1}$ ?

Problem 4. Can Theorems 1 and 2 be generalized to larger cardinals?

2. Proof of Theorem 1. The Boolean algebra of Theorem 1 will be obtained as the union of a sequence $\left\langle B_{\alpha}: \alpha<\omega_{1}\right\rangle$ of countable atomless fields of subsets of $\omega$. For each $\alpha, B_{\alpha+1}$ will be generated by $B_{\alpha}$ together with a single subset $x_{\alpha}$ of $\omega$, and if $\alpha$ is a limit ordinal then $B_{\alpha}$ will be the union of the preceding $B_{\beta}$ 's. The proposition $\diamond$ will be used to ensure that every potential uncountable chain or antichain is considered at some point.

The only difficulty lies in showing that if a countable set $M$ of maximal chains and antichains in $B_{\alpha}$ is specified, then $x_{\alpha}$ can be chosen so that every element of $M$ remains maximal in $B_{\alpha+1}$. This is done in Lemma 2.6. The set $x_{\alpha}$ is constructed essentially by a forcing argument, as the reader familiar with forcing will see, but no knowledge of forcing is necessary to follow the proof.

Given a partial ordering $(P, \leqslant)$, a set $D \subseteq P$ is called dense in $P$ if $\forall p \in P \exists q \in D q \leqslant p$.

For the purpose of the following lemmas, $B$ is always a countable atomless field of subsets of $\omega$. We shall be interested in the partial ordering

$$
P=\{(a, b): a, b \in B, a \subseteq b, b-a \neq 0\},
$$

where $\left(a_{1}, b_{1}\right) \leqslant\left(a_{2}, b_{2}\right)$ iff $a_{2} \subseteq a_{1}$ and $b_{1} \subseteq b_{2}$.
LEMMA 2.1. Let $m$ be a maximal antichain in $B$, and let $D_{1}(m)=\{(a, b) \in P: \forall x \subseteq \omega$ if $a \subseteq x \subseteq b$ then $x$ is comparable with some element of $m\}$. Then $D_{1}(m)$ is dense in $P$.

Proof. Let $(a, b) \in P$. Since $B$ is atomless there are disjoint non-empty $a_{1}, a_{2} \in B$ such that $a_{1} \cup a_{2}=b-a$. Let $c \in m$ be such that $a \cup a_{1}$ is comparable with $c$. If $c \subseteq a \cup a_{1}$, then $\left(a \cup a_{1}, b\right) \in D_{1}(m)$. If $a \cup a_{1} \subseteq c$, then $\left(a, a \cup a_{1}\right) \in D_{1}(m)$.

LEMMA 2.2. Let $m$ be a maximal chain in $B$, and let $D_{2}(m)=\{(a, b) \in P: \forall x \subseteq \omega$ if $a \subseteq x \subseteq b$ then $x$ is incomparable with some element of $m\}$. Then $D_{2}(m)$ is dense in $P$.

Proof. Let $(a, b) \in P$. Since $B$ is atomless, there are disjoint non-empty $a_{1}, a_{2}, a_{3} \in B$ such that $a_{1} \cup a_{2} \cup a_{3}=b-a$. If $a \cup a_{1} \in m$ then $\left(a \cup a_{2}, b-a_{1}\right)$ $\in D_{2}(m)$. If $a \cup a_{1} \notin m$ then there is $c \in m$ such that $c$ and $a \cup a_{1}$ are incomparable. If $c \cap\left(a_{2} \cup a_{3}\right)=0$ then $\left(a \cup a_{1}, b\right) \in D_{2}(m)$. If $c \cap a_{2} \neq 0$ then $\left(a \cup a_{1}, b-a_{2}\right)$ $\in D_{2}(m)$, and if $c \cap a_{3} \neq 0$ then $\left(a \cup a_{1}, b-a_{3}\right) \in D_{2}(m)$.

LEMMA 2.3. Let $m$ be a maximal antichain in $B$, and let $e, f \in B$. Let $D_{1}(m, e, f)$ $=\{(a, b) \in P: \forall x \subseteq \omega$ if $a \subseteq x \subseteq b$ then $(e \cap x) \cup(f-x)$ is comparable with some element of $m\}$. Then $D_{1}(m, e, f)$ is dense in $P$.

Proof. Let $(a, b) \in P$. It is easy to see that there is $\left(a^{\prime}, b^{\prime}\right) \leqslant(a, b)$ such that one of the following holds.
(1) $b^{\prime}-a^{\prime} \subseteq \omega-(e \cup f)$,
(3) $b^{\prime}-a^{\prime} \subseteq e-f$,
(2) $b^{\prime}-a^{\prime} \subseteq e \cap f$

If (1) or (2) holds, then clearly $\forall x \subseteq \omega$ if $a^{\prime} \subseteq x \subseteq b^{\prime}$ then $(e \cap x) \cup(f-x) \in B$, so $\left(a^{\prime}, b^{\prime}\right) \in D_{1}(m, e, f)$.

If (3) or (4) holds, then let $c=\left(e \cap a^{\prime}\right) \cup\left(f-b^{\prime}\right)$ and let $d=\left(e \cap b^{\prime}\right) \cup\left(f-a^{\prime}\right)$. Then $d-c \neq 0$ so $(c, d) \in P$. By Lemma 2.1 there is $\left(c^{\prime}, d^{\prime}\right) \in D_{1}(m)$ such that $\left(c^{\prime}, d^{\prime}\right) \leqslant(c, d)$. If (3) holds, then $\left(a^{\prime} \cup\left(c^{\prime}-c\right), b^{\prime}-\left(d-d^{\prime}\right)\right) \in D_{1}(m, e, f)$, while if (4) holds, then $\left(a^{\prime} \cup\left(d-d^{\prime}\right), b^{\prime}-\left(c^{\prime}-c\right)\right) \in D_{1}(m, e, f)$.

Lemma 2.4. Let $m$ be a maximal chain in $B$, and let $e, f \in B$. Let $D_{2}(m, e, f)$ $=\{(a, b) \in P: \forall x \subseteq \omega$ if $a \subseteq x \subseteq b$ then $(e \cap x) \cup(f-x)$ either lies in $m$ or else is incomparable with some element of $m\}$. Then $D_{2}(m, e, f)$ is dense in $P$.

Proof. Like Lemma 2.3, but using Lemma 2.2 instead of Lemma 2.1.

LBMMA 2.5. Let $c \in B$, and let $D_{3}(c)=\{(a, b) \in P: \forall x \subseteq \omega$ if $a \subseteq x \subseteq b$ then $x \neq c\}$.

Proof. By Lemma 2.2, letting $m$ be a maximal chain containing $c$.

LEMMA 2.6. Let $M$ be a countable collection of maximal chains and antichains. in $B$. Then for any $(a, b) \in P$ there is $x \notin B$ such that $a \subseteq x \subseteq b$ and if $B^{\prime}$ is the field of sets generated by $B \cup\{x\}$, then every element of $M$ remains maximal in $B^{\prime}$.

Proof. Let $D_{0}, D_{1}, \ldots$ enumerate all sets of the form $D_{1}(m, e, f), D_{2}(m, e, f)$, and $D_{3}(c)$ where $m \in M$ and $c, e, f \in B$. Form a sequence $\left\langle\left(a_{n}, b_{n}\right): n \in \omega\right\rangle$ as follows: Let $a_{0}=a, b_{0}=b$. Given $\left(a_{n}, b_{n}\right)$, let $\left(a_{n+1}, b_{n+1}\right) \in D_{n}$ be such that $\left(a_{n+1}, b_{n+1}\right)$ $\leqslant\left(a_{n}, b_{n}\right)$. Let $x=\bigcup\left\{a_{n}: n \in \omega\right\}$. Note that every element of $b^{\prime}$ has the form $(e \cap x) \cup(f-x)$ for some $e, f \in B$. But now by Lemmas 2.3-2.5 it is clear that 
$x \notin B$ and every element of $M$ remains maximal in $B^{\prime}$. It is easy to see that $B^{\prime}$ is atomless.

Proof of Theorem 1. Let $\left\langle S_{\alpha}: \alpha<\omega_{1}\right\rangle$ be a $\rangle$-sequence. Since $<$ implies the continuum hypothesis, there is an enumeration $\left\langle a_{\alpha}: \alpha<\omega_{1}\right\rangle$ of the power set of $\omega$ in which each element occurs uncountably many times.

By induction, we construct a sequence $\left\langle B_{\alpha}: \alpha\left\langle\omega_{1}\right\rangle\right.$ of countable atomless fields of subsets of $\omega$, and a sequence $\left\langle M_{\alpha}: \alpha<\omega_{1}\right\rangle$ where $M_{\alpha}$ is a finite or countably infinite set of maximal chains and antichains in $B_{\alpha}$.

Let $B_{0}$ be arbitrary, and let $M_{0}=0$. If $\alpha$ is a limit ordinal, let $B_{\alpha}=\bigcup\left\{B_{\beta}: \beta<\alpha\right\}$ and $M_{\alpha}=\bigcup\left\{M_{\beta}: \beta<\alpha\right\}$. Finally, suppose $\alpha=\beta+1$. If $\left\{a_{\xi}: \xi \in S_{\beta}\right\}$ is a maximal chain or antichain in $B_{\beta}$ then let $M_{\beta+1}=M_{\beta} \cup\left\{\left\{\alpha_{\xi}: \xi \in S_{\beta}\right\}\right\}$; otherwise let $M_{\beta+1}=M_{\beta}$. Choose $x_{\beta} \notin B_{\beta}$ by Lemma 2.6 so that if $B_{\beta+1}$ is generated by $B_{\beta} \cup\left\{x_{\beta}\right\}$ then every chain or antichain in $M_{\beta+1}$ remains maximal in $B_{\beta+1}$. Moreover, if $a_{\beta} \in B_{\beta}$ then we may assume $x_{\beta} \subseteq a_{\beta}$.

Let $B=\bigcup\left\{B_{\alpha}: \alpha<\omega_{1}\right\}$. It is clear that every nonzero element of $B$ is uncountable. Suppose $m \subseteq B$ is a maximal chain. Let $S=\left\{\alpha: a_{\alpha} \in m\right\}$. Then it is easy to see that $\left\{\alpha:\left\{a_{\beta}: \beta \in S \cap \alpha\right\}\right.$ is a maximal chain in $\left.B_{\alpha}\right\}$ is closed and unbounded. Since $\left\{\alpha: S_{\alpha}=S \cap \alpha\right\}$ is stationary there is $\alpha$ such that $S_{\alpha}=S \cap \alpha$ and $\left\{a_{\beta}: \beta \in S_{\alpha}\right\}$ is a maximal chain in $B_{\alpha}$. But then $\left\{a_{\beta}: \beta \in S_{\alpha}\right\} \in M_{\gamma}$ for every $\gamma \geqslant \alpha$ so $\left\{a_{\beta}: \beta \in S_{\alpha}\right\}$ is maximal in $B$. Hence $m=\left\{a_{\beta}: \beta \in S_{\alpha}\right\}$, and $m$ is countable. It can be shown similarly that all antichains are countable. This completes the proof.

3. Proof of Theorem 2. We will obtain our Boolean algebra as the union of a sequence $\left\langle B_{\alpha}: \alpha<\omega_{1}\right\rangle$ as before, but in addition we will construct a sequence $\left\langle I_{\alpha}: \alpha<\omega_{1}\right\rangle$, where $I_{\alpha}$ is a maximal ideal in $B_{\alpha}$ and $I_{\alpha} \subseteq I_{\beta}$ whenever $\alpha \leqslant \beta$. It will turn out that $U\left\{I_{\alpha}: \alpha<\omega_{1}\right\}$ is the maximal ideal of countable elements in the Boolean algebra.

In Lemmas 3.1-3.7, $B$ will always be a countable atomless field of subsets of $\omega$, and $I$ will be a maximal ideal in $B$. The partial ordering $P$ to be used this time is $P=\{(a, b): a \in I, b \in B-I$, and $a \subseteq b\}$, ordered as before, i.e., $\left(a_{1}, b_{1}\right) \leqslant\left(a_{2}, b_{2}\right)$ iff $a_{1} \supseteq a_{2}$ and $b_{1} \subseteq b_{2}$.

Lemma 3.1. Let $m \subseteq I$, and let $D_{1}(m)=\{(a, b) \in P$ : either $\forall c \in m \quad c \nsubseteq b$ or $\exists c \in m \quad c \subseteq a\}$. Then $D_{1}(m)$ is dense in $P$.

Proof. Let $(a, b) \in P$. If $(a, b) \notin D_{1}(m)$, then $\exists c \in m c \subseteq b$. But then $(a \cup c, b)$ $\in D_{1}(m)$.

LEMMA 3.2. Let $c \in B$ and let $D_{2}(c)=\{(a, b) \in P: \forall x \subseteq \omega$ if $a \subseteq x \subseteq b$ then $x \neq c\}$. Then $D_{2}(c)$ is dense in $P$.

Lemma 3.3. Let $c \in I$ and let $D_{3}(c)=\{(a, b) \in P: c \subseteq a \cup(\omega-b)\}$. Then $D_{3}(c)$ is dense in $P$.

Lemma 3.4. Suppose $D$ is dense in $P$. Let $S(D)=\{(a, b) \in P:(\omega-b, \omega-a) \in D\}$. Then $S(D)$ is dense in $P$.
Lemma 3.5. Suppose $D$ is dense in $P$ and $e, f \in I$. Let

$$
T(D, e, f)=\{(u, b) \in P:((a-e) \cup f,(b-e) \cup f) \in D\} .
$$

Then $T(D, e, f)$ is dense in $P$.

Proof. Let $(a, b) \in P$. By Lemma 3.3 there is $\left(a^{\prime}, b^{\prime}\right) \leqslant(a, b)$ such that $e \cup f \subseteq a^{\prime} \cup\left(\omega-b^{\prime}\right)$. Let $e^{\prime}=a^{\prime} \cap e, f^{\prime}=a^{\prime} \cap f$. Find $(x, y) \in D$ such that $(x, y) \leqslant\left(\left(a^{\prime}-e\right) \cup f,\left(b^{\prime}-e\right) \cup f\right)$. Now let $a^{\prime \prime}=(x-(e \cup f)) \cup e^{\prime} \cup f^{\prime}$, and $b^{\prime \prime}=(y-(a \cup f)) \cup e^{\prime} \cup f^{\prime}$. Then it is easy to see $\left(a^{\prime \prime}, b^{\prime \prime}\right) \in T(D, e, f)$.

Now suppose $M$ is a countable (or finite) collection of subsets of $B$, and let $\mathscr{Z}$ be the smallest collection of dense sets such that

(a) every set of the form $D_{1}(m), D_{2}(c), D_{3}(e)$, for $m \in M, c \in B, e \in I$, lies in $\mathscr{D}$, and

(b) if $D \in \mathscr{D}$ and $e, f \in I$, then $S(D), T(D, e, f) \in \mathscr{D}$.

Let us call $x \subseteq \omega(M, I)$-generic over $B$ iff $\forall D \in \mathscr{D} \exists(a, b) \in D \quad a \subseteq x \subseteq b$. Since $\mathscr{L}$ is countable it is clear that there exists $x \subseteq \omega$ which is $(M, I)$-generic over $B$.

LEMMA 3.6. Let $x$ be $(M, I)$-generic over $B$, and let $B^{\prime}$ be the field of sets generated by $B \cup\{x\}$. Then every element of $B^{\prime}-B$ is $(M, I)$-generic over $B$.

Proof. It is evident from condition (b) above and Lemmas 3.4 and 3.5 that if $x$ is $(M, I)$-generic over $B$, then so are $(x-e) \cup f$ and $\omega-x$, if $e, f \in I$. To complete the proof, it will suffice to show that any element of $B^{\prime}-B$ has the form $(x-e) \cup f$ or $((\omega-x)-e) \cup f$ for some $e, f \in I$.

First note that if $x$ is $(M, I)$-generic over $B$, then for any $a \in I$, both $a \cap x$ and $a-x$ are in $I$. This follows immediately trom Lemma 3.3 .

We observed in the last section that every element of $B^{\prime}-B$ has the form $(e \cap x) \cup(f-x)$ for some $e, f \in B$. But by genericity of $x$ and $\omega-x$, the remark in the preceding paragraph shows that we cannot have both $e, f \in I$, or both $e, f \notin I$, since otherwise $(e \cap x) \cup(f-x) \in B$.

If $f \in I$ and $e \notin I$ then $(e \cap x) \cup(f-x)=(x-(\omega-e)) \cup(f-x)$, where both $\omega-e$ and $f-x$ are in $I$. If $e \in I$ and $f \notin I$ then $(e \cap x) \cup(f-x)=((\omega-x)-(\omega-f)) \cup$ $\cup(e \cap x)$ where $\omega-f$, e $\cap x \in I$, and we are done.

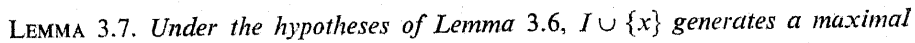
ideal $I^{\prime}$ in $B^{\prime}$ and $\forall a \in I \forall b \in B^{\prime}$ if $b \subseteq a$ then $b \in B$.

Proof. We check the last assertion first. If $b \in B^{\prime}-B$, then $b$ is $(M, I)$-generic over $B$, so as observed in the preceding proof, if $a \in I$ then $a \cap b \in I$, so we cannot have $b \subseteq a$.

For the rest, suppose $x \cup a=\omega$ for some $a \in I$. Then $x=\omega-(a-x)$, and since $a-x \in I$, we would have $x \in B$, contradiction. Hence $I^{\prime}$ is a proper ideal. It remains only to check maximality. Let $y \in B^{\prime}-B$. If $y=(x-e) \cup f$ for some $e, f \in I$ then $y \in I$. If not, then $y=((\omega-x)-e) \cup f$ for $e, f \in I$. But then $\omega-y=(x-f) \cup$ $\cup(e-f) \in I$ 
Pro of of Theorem 2. Let $\left\langle S_{\alpha}: \alpha \in \omega_{1}\right\rangle$ be a $\vartheta$-sequenct, and let $\left\langle A_{\alpha}: \alpha<\omega_{1}\right\rangle$ enumerate the power set of $\omega$. For each $\beta<\omega_{1}$, let $m_{\beta}=\left\{a_{\alpha}: \alpha \in S_{\beta}\right\}$.

We obtain sequences $\left\langle B_{\alpha}: \alpha<\omega_{1}\right\rangle,\left\langle I_{\alpha}: \alpha<\omega_{1}\right\rangle$, and $\left\langle M_{\alpha}: \alpha<\omega_{1}\right\rangle$ by induction as follows: Let $B_{0}$ be an arbitrary countable atomless field of subsets of $\omega$, and let $I_{0}$ be a maximal ideal in $B_{0}$. Given $B_{\alpha}$ and $I_{\alpha}$, a maximal ideal in $B_{\alpha}$, let $M_{\alpha}=\left\{m_{\beta}: \beta \leqslant \alpha\right.$ and $\left.m_{\beta} \subseteq B_{\alpha}\right\}$. Let $x_{\alpha}$ be $\left(M_{\alpha}, I_{\alpha}\right)$-generic over $B_{\alpha}$ and let $B_{\alpha+1}$ be generated by $B_{\alpha} \cup\left\{x_{\alpha}\right\}$, and let $I_{\alpha+1}$ be generated by $I_{\alpha} \cup\left\{x_{\alpha}\right\}$. If $\alpha$ is a limit ordinal, let $B_{\alpha}=\bigcup\left(B_{\beta}: \beta<\alpha\right\}$, and $I_{\alpha}=\bigcup\left\{I_{\beta}: \beta<\alpha\right\}$. Let $B=\bigcup\left\{B_{\alpha}: \alpha<\omega_{1}\right\}$ and $I=\bigcup\left\{I_{\alpha}: \alpha<\omega_{1}\right\}$.

It is easy to see that each $B_{\alpha}$ is atomless so $B$ is an atomless field of subsets of $\omega$. It follows from Lemma 3.7 that $I$ is a maximal ideal of countable elements of $B$. It remains only to show that $B$ has no uncountable antichains.

Suppose $m \subseteq I$ were an uncountable antichain. Let $S=\left\{\alpha: a_{x} \in m\right\}$. Let $Z=\left\{\alpha:\left\{a_{\beta}: \beta \in S \cap \alpha\right\}=m \cap B_{\alpha} \quad\right.$ and $\quad\left(\forall b \in B_{\alpha}-I_{\alpha}\right)$ if $\exists c \in m \quad c \subseteq b$ then $\left.(\exists \beta \in S \cap \alpha) a_{\beta} \subseteq b\right\}$. It is easy to see that $Z$ is closed and unbounded. Hence there is $x \in Z$ such that $S_{\alpha}=S \cap \alpha$.

We assert that $m=m_{\alpha}$. This will show that there are no uncountable antichains included in $I$, and hence that there are no uncountable antichains in $B$ (since if $m \subseteq B-I$ were an antichain, so would be $\{\omega-a: a \in m\} \subseteq I)$.

Clearly $m_{\alpha} \subseteq m$. For each $c \in B-B_{0}$, let $\varrho(c)$ be the least ordinal $\beta$ such that $c \in B_{\beta+1}-B_{\beta}$. Suppose there exists $c \in m-m_{\alpha}$. Choose such a $c$ with $\varrho(c)$ minimal. Let $\varrho(c)=\beta \geqslant \alpha$. Then $c$ is $\left(M_{\beta}, I_{\beta}\right)$-generic over $B_{\beta}$ so since $m_{\alpha} \in M_{\beta}$ there exists $(a, b) \in D_{1}\left(m_{\alpha}\right)$ (computed in $\left.B_{\beta}\right)$ such that $a \subseteq c \subseteq b$. Since $c$ is not comparable with any element of $m_{\alpha}$ it must be the case that $\forall c^{\prime} \in m_{\alpha} c^{\prime} \nsubseteq b$. We may assume that $\varrho(b)$ is minimal for $b \in B_{\beta}$ such that $a \subseteq c \subseteq b$ and $\forall c^{\prime} \in m_{\alpha} \cdot c^{\prime} \nsubseteq b$.

Now $\varrho(b)>\alpha$ since otherwise $\alpha \in Z$ implies that $\forall c^{\prime} \in m c^{\prime} \nsubseteq b$, contradiction. Say $\varrho(b)=\gamma \geqslant \alpha$. Then $b$ is $\left(M_{\gamma}, I_{\gamma}\right)$-generic over $B_{\gamma}$, and there must be $\left(a^{\prime}, b^{\prime}\right)$ $\dot{\in} D_{1}\left(m_{\alpha}\right)$ (computed in $B_{\gamma}$ ) such that $a^{\prime} \subseteq b \subseteq b^{\prime}$. But then clearly we must have $\forall c^{\prime} \in m_{\alpha} c^{\prime} \nsubseteq b^{\prime}$ and $\varrho\left(b^{\prime}\right)<\gamma$, contradicting minimality of $\varrho(b)$. Hence $B$ has no uncountable antichains.

It follows that $B$ has no uncountable chains, for if $m$ were an uncountable chain we could, as above, assume that $m \subseteq I$. But since all the elements of $/$ are countable, $m$. must have a subset well-ordered in type $\omega_{1}$, and this contradicts the fact that $B$ has no uncountable antichains.

Added in proof December 3, 1980. Most of the problems have been solved. Shelah found an uncountable algebra with no uncountable chains or antichains using only $\mathrm{CH}$; the first author showed it consistent that every uncountable algebra has an uncountable antichain; Shetah and Van Wesep independently answered Problem 3 negatively; and Shelah has generalized the -style constructions to larger cordinals.

\section{References}

[1] E.'S. Berney, preprint.

[2] K. Devlin, Aspects of Constructibility, Lecture Notes in Mathematics, vol. 354
13] P. Erdös, A. Hajnal, A. Máté and R. Rado, Combinatorial Set Theory: Partition Relations for Cardinals, manuscript.

4] G. Fodor, Eine Bemerkung zur Theorie der regressiven Funktionen, Acta Sci. Math. 17 (1956), pp. 139-142.

[5] A. Hajnal, Proof of a conjecture of $S$. Ruziewicz, Fund. Math. 50 (1961), pp. 123-128.

[6] K. Kunen, mimeographed notes.

[7] - and F. Tall, Between Martin's Axiom and Souslin's Hypothesis, preprint.

\section{DARTMOUTH COLLEGE}

Hanover, New Hampshire

EÖTVÖS LORAND UNIVERSTTY

Budapest

Accepté par la Rédaction le 26. 10. 1978 